\title{
ON TYPICALLY-REAL FUNCTIONS IN A CUT PLANE
}

\author{
BY E. P. MERKES
}

1. Introduction. Let

$$
w=f(z)=z+a_{2} z^{2}+\cdots+a_{n} z^{n}+\cdots
$$

be regular in $|z|<1$ and real valued if and only if $z$ is real and $-1<z<1$. Then $f(z)$ is said to be typically-real in the unit circle. Rogosinski [3] has shown that a necessary and sufficient condition for a regular function $f(z)$ in $|z|<1$, where $f(0)=0, f^{\prime}(0)=1$, to be typically-real is that

$$
f(z)=\frac{z}{1-z^{2}} \phi(z), \quad \phi(0)=1,
$$

where $\phi(z)$ is regular, real for real $z$, and has positive real part for $|z|<1$. Furthermore, if $w=f(z)$ maps each circle $|z|=r<1$ into a contour having the property that every line parallel to the imaginary axis cuts this contour in at most two points, then $f(z)$ is said to be convex in the direction of the imaginary axis. A necessary and sufficient condition that $f(z)$ be convex in the direction of the imaginary axis is that $z f^{\prime}(z)$ be typically-real in the unit circle $[1 ; 2]$.

It is the main purpose of this paper to display a connection between typically-real functions on one hand and Stieltjes transforms and continued fractions on the other. To this end consider first a function $F(\zeta)$ which is real valued for real $\zeta$ and regular in the complex plane cut along the negative real axis from $-\infty$ to -1 . If, further, $F(0)=0$, $F^{\prime}(0)=1$, and $\operatorname{Im} F(\zeta) \neq 0$ for nonreal values of $\zeta$ in this cut plane, the function $F(\zeta)$ is said to be typically-real in the cut plane. The class of all such functions $F(\zeta)$ is denoted by $T[-\infty,-1]$. On the other hand, if $F(0)=1$ and $\operatorname{Re} F(\zeta)>0$ for $\zeta$ in this cut plane, we say that $F(\zeta)$ is in the class $R[-\infty,-1]$.

If the usual agreements are made regarding the normalization, a similar definition can be given for a function to be typically-real in the complex plane cut along the real axis from $a$ to $b$ such that the cut does not include both 0 and $\infty$. In each case there is a linear fractional transformation with real coefficients which carries such a function into one which, except for the normalization, is in the class $T[-\infty,-1]$. For this reason attention is confined to the latter class.

Presented to the Society, January 22, 1959; received by the editors November 28. 1958 and, in revised form, February 19, 1959. 
2. Some characterizations. The univalent transformation

$$
\zeta=\frac{4 z}{(1-z)^{2}}
$$

maps the disc $|z|<1$ onto the $\zeta$-plane cut along the negative real axis from $\infty$ to -1 . Since $\operatorname{Im} \zeta=4\left(1-|z|^{2}\right)^{-1} \operatorname{Im} z$, the function

$$
f(z)=\frac{1}{4} F\left[\frac{4 z}{(1-z)^{2}}\right]
$$

is typically-real in the unit circle whenever $F(\zeta)$ is in the class $T[-\infty,-1]$ and conversely. Thus there is a one-to-one correspondence between the class $T[-\infty,-1]$ and the class of typically-real functions in the unit circle.

By (1.2) and (2.2) we obtain, after the change of variable (2.1), the following restatement of the cited theorem of Rogosinski:

Theorem 2.1. $F(\zeta)$ is in $T[-\infty,-1]$ if and only if there exists a function $\Phi(\zeta)$ in the class $R[-\infty,-1]$ such that

$$
F(\zeta)=\frac{\zeta}{(1+\zeta)^{1 / 2}} \Phi(\zeta)
$$

where that branch of $(1+\zeta)^{1 / 2}$ is chosen in the cut plane which is positive at $\zeta=0$.

It is known [6, p. 278] that $\Phi(\zeta)$ is in $R[-\infty,-1]$ if and only if there exists a nondecreasing function $\alpha(t)$ on $0 \leqq t \leqq 1$ such that $\alpha(1)$ $-\alpha(0)=1$ and

$$
\Phi(\zeta)=(1+\zeta)^{1 / 2} \int_{0}^{1} \frac{d \alpha(t)}{1+\zeta t}
$$

Thus we have the following result:

Corollary 2.1. A necessary and sufficient condition for $F(\zeta)$ to be in $T[-\infty,-1]$ is that there exists a nondecreasing function $\alpha(t)$ on $0 \leqq t \leqq 1$ such that $\alpha(1)-\alpha(0)=1$ and

$$
F(\zeta)=\int_{0}^{1} \frac{\zeta d \alpha(t)}{1+\zeta t}
$$

By well-known [6, p. 263] relations between the Hausdorff moment problem and $S$-fractions, the following corollaries are obtained from the above: 
COROllary 2.2. $F(\zeta)$ is in $T[-\infty,-1]$ if and only if

$$
F(\zeta)=\frac{\zeta}{1}+\frac{\left(1-g_{0}\right) g_{1} \zeta}{1}+\cdots+\frac{\left(1-g_{n}\right) g_{n+1} \zeta}{1}+\cdots
$$

where $0 \leqq g_{n} \leqq 1, n=0,1,2, \cdots$.

Corollary 2.3. Let

$$
F(\zeta)=\zeta-\alpha_{2} \zeta^{2}+\alpha_{3} \zeta^{3}-\cdots+(-1)^{n+1} \alpha_{n} \zeta^{n}+\cdots,
$$

Then $F(\zeta)$ is in $T[-\infty,-1]$ if and only if the sequence $1, \alpha_{2}, \alpha_{3}, \cdots$, $\alpha_{n}, \cdots$ is totally monotone.

By (2.1), (2.2), and (2.6) we obtain, after an equivalence transformation, the following continued fraction characterization of this class:

Theorem 2.2. A necessary and sufficient condition for $f(z)$ to be typically-real in the unit circle is that $f(z)$ have a continued fraction expansion of the form

$$
f(z)=\frac{z}{1-z}\left[\frac{1}{1-z}+\frac{4\left(1-g_{0}\right) g_{1} z}{1-z}+\cdots\right.
$$

$$
\left.+\frac{4\left(1-g_{n}\right) g_{n+1} z}{1-z}+\cdots\right]
$$

where $0 \leqq g_{n} \leqq 1, n=0,1,2, \cdots$.

The correspondence between the classes under consideration can also be used to characterize typically-real functions in $|z|<1$ in terms of Schur summability. For this purpose let $g(z)=\sum_{j=0}^{\infty} c_{j}(-z)^{i}$, $G(\zeta)=\sum_{j=0}^{\infty} \gamma_{j}(-\zeta)^{i}$ be related by

$$
\frac{1}{2}(1-z) g(z)=G(\zeta), \quad \zeta=4 z /(1-z)^{2} .
$$

The transformation from the sequence $s_{n}=\sum_{j=0}^{n} c_{j}$ to the sequence $S_{n}=\sum_{j=0}^{n} \gamma_{j}$ by means of the identity (2.9) is called the $(S)$-transformation. The sequence $\left\{s_{n}\right\}$ is said to be $(S)$-summable to the value $S$ if $S=\lim _{n \rightarrow \infty} S_{n}$. Scott and Wall [4] have introduced and studied in detail this consistent method of summability.

THEOREM 2.3. Let $f(z)=\sum_{j=0}^{\infty} a_{j} z^{j}, a_{0}=0, a_{1}=1$, be regular and real for real $z$ in the unit circle. Then $f(z)$ is typically-real in $|z|<1$ if 
and only if the sequence $\left\{1+(-1)^{n}\left(a_{n+2}-a_{n}\right) / 2\right\}_{n=0}^{\infty}$ is $(S)$-summable to a value not exceeding unity and such that $\left\{S_{n}-S_{n-1}\right\}_{n=0}^{\infty}$, where $S_{-1}=0$ and the sequence $S_{n}$ is the $(S)$-transform of the given sequence, is totally monotone.

Proof. By (2.4) and the characterization of Rogosinski $f(z)$ is typically-real in the unit circle if and only if

$$
\begin{aligned}
& \frac{(1-z)^{2}}{4 z}\left[\frac{(1+z)^{2}}{z} f(z)-1\right]=\frac{(1-z)^{2}}{4 z}\left[\frac{1+z}{1-z} \phi(z)-1\right] \\
(2.10) \quad= & \frac{1}{\zeta}\left[(1+\zeta)^{1 / 2} \Phi(\zeta)-1\right]=\frac{1}{\zeta}\left[(1+\zeta) \int_{0}^{1} \frac{d \alpha(t)}{1+\zeta t}-1\right] \\
& =\int_{0}^{1} \frac{(1-t) d \alpha(t)}{1+\zeta t}
\end{aligned}
$$

where $\zeta=4 z /(1-z)^{2}, \Phi(\zeta)=\phi(z)$, and $\alpha(1)-\alpha(0)=1$. Let $G(\zeta)$ be the function represented by the integral in (2.10). Then $\left\{\gamma_{j}\right\}$ is totally monotone and $\sum_{j=0}^{\infty} \gamma_{j} \leqq 1$ where $G(\zeta)=\sum_{j=0}^{\infty} \gamma_{j}(-\zeta)^{j}$ for $|\zeta|<1$ [6, p. 284]. Moreover, if the left-hand side of (2.10) is taken to be the function $(1-z) g(z) / 2$ in (2.9), it is easily seen that $s_{n}=\sum_{j=0}^{n} c_{j}$ $=1+(-1)^{n}\left(a_{n+2}-a_{n}\right) / 2$. The theorem now follows at once from the definition of $(S)$-summability.

3 . Some properties of the class $T[-\infty,-1]$. Among other considerations the following theorem yields some properties of $S$-fractions of the form (2.6):

THEOREM 3.1. If $F(\zeta)$ is in $T[-\infty,-1]$, then it is univalent for $\operatorname{Re} \zeta>-1$. This result is sharp. Moreover, $F(\zeta)$ is convex in the direction of the imaginary axis for $|\zeta|<1$.

The domain of univalence, $\operatorname{Re} \zeta>-1$, for the class is obtained by a trivial adjustment of a proof given by Thale [5, p. 233]. That the result is sharp is established by considering the following functions of $T[-\infty,-1]$ :

$$
F(\zeta ; c)=\frac{\zeta(1+c \zeta)}{1+\zeta}, \quad 0<c<1 .
$$

It is easily seen that for any two distinct points $\zeta_{1}$ and $\zeta_{2}$ in the upper (lower) half-plane and on the line $\operatorname{Re} \zeta=-1$ there is a constant $c_{0}$, $0<c_{0}<1$, such that $F\left(\zeta_{1} ; c_{0}\right)=F\left(\zeta_{2} ; c_{0}\right)$.

In order to prove that $F(\zeta)$ is convex in the direction of the imaginary axis, note that by $(2.5)$ 


$$
\operatorname{Im}\left[\zeta F^{\prime}(\zeta)\right]=\operatorname{Im} \zeta \int_{0}^{1} \frac{1-|\zeta|^{2} t^{2}}{|1+\zeta|^{4}} d \alpha(t)
$$

It immediately follows that $\zeta F^{\prime}(\zeta)$ is typically-real. This yields the desired result.

It is interesting to note [5] that the domain of univalence for the class $T[-\infty,-1]$ in Theorem 3.1 is also a sharp domain of univalence for functions of the form $F(\zeta) / \zeta$, where $F(\zeta)(\not \equiv \zeta)$ is in $T[-\infty,-1]$. One way to see this is to observe that by $(2.7)$

$$
F(\zeta) / \zeta=1-\alpha_{2} F_{1}(\zeta)
$$

By a property of totally monotone sequences it now follows from Corollary 2.3 that $F_{1}(\zeta)$ is in $T[-\infty,-1]$. The univalence of $F_{1}(\zeta)$ then implies that of $F(\zeta) / \zeta$.

4. A domain of univalence for typically-real functions in the unit circle. Let $f(z)$ be typically-real in the unit circle. By the one-to-one correspondence of $\S 2$ there exists a function $F(\zeta)$ in $T[-\infty,-1]$ such that (2.2) holds. From Theorem 3.1 and the fact that (2.1) is a univalent transformation, we conclude that $f(z)$ is univalent for

$$
\operatorname{Re}\left[\frac{4 z}{(1-z)^{2}}\right]>-1
$$

The region (4.1) can be expressed in the form given in the following result:

THEOREM 4.1. If $f(z)$ is typically-real in the unit circle, then $f(z)$ is univalent for $z$ in the domain $D$ bounded by the circular arcs $z=r e^{i \theta}$, where

$$
r=\left(1+\sin ^{2} \theta\right)^{1 / 2}-|\sin \theta|, \quad 0 \leqq \theta<2 \pi .
$$

The result is sharp in the sense that any open region of univalence for this class of functions which contains $D$ is coincident with $D$.

The sharpness result of the theorem follows from that for the class $T[-\infty,-1]$.

The largest circular domain with center at the origin and contained in the domain $D$ has radius $(2)^{1 / 2}-1$.

Corollary 4.1. If $f(z)$ is typically-real in the unit circle, then $f(z)$ is univalent in the disc $|z|<(2)^{1 / 2}-1$. This circular domain of univalence cannot be replaced by a larger circular domain with center at the origin in which each function of the class under consideration is univalent.

The function 


$$
f(z)=z\left(1+z^{2}\right) /\left(1-z^{2}\right)^{2}
$$

is typically-real in the unit circle. Moreover, since the derivative of this function vanishes at $z= \pm\left((2)^{1 / 2}-1\right) i,(4.3)$ is not univalent in any larger circular domain with center at the origin than that given in Corollary 4.1.

\title{
REFERENCES
}

1. L. Fejér, Neue Eigenschaften der Mittelwerte bei den Fourierreihen, J. London Math. Soc. vol. 8 (1933) pp. 53-62.

2. M. S. Robertson, On the coefficients of a typically-real function, Bull. Amer. Math. Soc. vol. 41 (1935) pp. 565-572.

3. W. Rogosinski, Über positive harmonische Entwicklungen und typischreelle Potenzreihen, Math. Z. vol. 35 (1932) pp. 93-121.

4. W. T. Scott and H. S. Wall, The transformation of series and sequences, Trans. Amer. Math. Soc. vol. 51 (1942) pp. 255-279.

5. J. S. Thale, Univalence of continued fractions and Stieltjes transforms, Proc. Amer. Math. Soc. vol. 7 (1956) pp. 232-244. 1948.

6. H. S. Wall, Analytic theory of continued fractions, New York, Van Nostrand,

De Paul University

\section{ON THE POLE AND ZERO LOCATIONS OF RATIONAL LAPLACE TRANSFORMATIONS OF NON-NEGATIVE FUNCTIONS}

\author{
ARMEN H. ZEMANIAN
}

Let $a(t)$ be a real, bounded function of the real variable $t$ defined in the interval, $0 \leqq t<\infty$, and let its Laplace-Stieltjes transform,

$$
F(s)=\int_{0}^{\infty} e^{-8 t} d a(t),
$$

be a rational function of the complex variable $s=\sigma+i \omega$, having at least as many poles as zeros. $F(s)$ may be written as

$$
F(s)=\frac{\prod_{i=1}^{h}\left(s-\eta_{i}\right) \prod_{i=1}^{n}\left(s-\nu_{i}\right)}{\prod_{i=1}^{m}\left(s-\rho_{i}\right) \prod_{i=1}^{q}\left(s-\xi_{i}\right)}
$$

where the $\eta_{i}$ and $\rho_{i}$ are real and the $\nu_{i}$ and $\xi_{i}$ are complex. Under these 1959.

Received by the editors December 22, 1958 and, in revised form, February 10, 